\title{
Phase Window Analysis of Partial Discharges in an Oil Pressboard Insulation System
}

\author{
K. Raja, M.N. Narayanachar and R.S. Nema \\ Department of High Voltage Engineering \\ Indian Institute of Science \\ Bangalore-560 012, India
}

\begin{abstract}
Partial discharge (PD) distributions have been studied in a representative oil-pressboard insulation system using a phase resolved PD analyzer built for the purpose [1]. The system divided a cycle of the applied voltage, starting from the zero of positive half cycle, into sixteen equal phase windows $\left(\Phi_{1}\right.$ to $\left.\Phi_{16}\right)$ to determine the discharge magnitude in each phase. PD data are recorded according to their phase of occurrence with respect to the power frequency cycle, their magnitude and number over a period of time. The effect of different test procedures, stress levels and its duration on the ensuing PD patterns have been statistically studied. The results of the analysis help in identifying the probable phase windows which have significant contribution towards overall aging and the ensuing $\mathrm{PI}^{\prime}$ distribution patterns.
\end{abstract}

\section{Experimental Details}

\section{Sample and Electrodes}

The sample-electrode assembly comprised of a circular pressboard sample of $2.1 \mathrm{~mm}$ thickness and $50 \mathrm{~mm}$ diameter, held centre symmetrical between two Rogowski profile uniform field electrodes. This insulation configuration simulate electric fields at the edges as in actual wedge shaped oil gaps commonly encountered in oil filled transformers between the rounded conductors and pressboard spacers. The entire assembly was placed in an air tight chamber filled with purified and degassed transformer oil. The breakdown voltage of oil was in the range of 40 to $45 \mathrm{kV}$ for a $1 \mathrm{~mm}$ gap between $12.5 \mathrm{~mm}$ spheres.

\section{Experimental Proced $;$ e}

It was oberved during preliminary experiments that PD spectrum depended upon the level of stress and its duration. The inception voltage $\left(\mathrm{V}_{i}\right)$ of the sample at which discharges first appeared and persisted for atleast 1 minute was in the range of $30 \mathrm{kV}$ to $37 \mathrm{kV}$. Depending upon the manner of voltage application, the experiments were classified into three separate test procedures which are as follows.

For test procedure 1 , after determining $\mathrm{V}_{i}$ the voltage across the sample was maintained at this level for about 3 to 6 hours. If the discharges observed on the oscilloscope were intermittent and if the number density and discharge magnitude (q) were insignificant, then the next higher step of voltage $\left(1.1 \mathrm{~V}_{i}\right)$ was applied. Subsequent steps were 5 percent higher than that of previous step, until the start of burst discharges ie. when the discharge magnitude and pulse rate suddenly increased and persisted in all phases. The voltage of this step was held constant till the sample failed.

In the second test procedure, the sample was aged at $1.1 \mathrm{~V}_{i}$ for about 3 to 6 hours after which the voltage was raised to $1.15 \mathrm{~V}_{i}$ and held constant till the sample failed.

For the third procedure, the voltage across the sample was held constant at $1.15 \mathrm{~V}_{i}$ till the sample failed.

\section{Experimental Results}

The results of aging experiments show that discharges in the wedge shaped gap, formed between electrodes and sample surface, commenced at $1.15 \mathrm{~V}_{i}$ and this observation coincided with the start of burst discharges, an event which marks the transition from a period of insignificant pulse rate and scanty distribution of pulses amongst different phases to a period of copious pulses in all phases with $\mathrm{q}$ ranging betweer. $3 \mathrm{pC}$ to $150 \mathrm{pC}$.

A comparative study of all aging experiments shows a common PD pattern for this insulation con- 
figuration, the results of which are summarized in Table 1, where in, total pulse count $\left(\mathrm{N}_{t}\right)$ of individual phases is expressed as a percentage value of integrated $N_{t}$ quantity of all phases in the full cycle. Similar patterns were observed when only phases in the positive or negative half cycle were considered alone. The same was true for the integrated charge quantity $\mathrm{Q}_{t}$.

Irrespective of test procedure, stress level and its duration it is clear that $\Phi_{14}$ is the single largest contributor with its contribution on the average between 32 to 38 percent. This is followed by either $\Phi_{16}$ for procedures $1 \& 2$ and $\Phi_{13}$ for procedure 3 and their contributions were about 21 and 13 percent. $\Phi_{7}$ followed by $\Phi_{8}$ were the least contributors and their shares were respectively less than 0.7 and 1.0 percent. Also, it was observed that $\mathrm{N}_{t}$ of negative half cycle is about 80 percent of total full cycle $\mathrm{N}_{t}$.

\section{Statistical Details and Results}

Prior to the start of burst discharges, the distribution of pulse amplitudes in individual phase windows were statistically insignificant in number for any distribution fitting of the spectrum. To characterize this aging period, maximum PD magintude $\left(Q_{m}\right)$ of the $P D$ data collected at regular intervals of time for different phases and full cycle were arranged as a sequence of time series records (TSR). Gumbel's extreme value distribution of the largest element [2] given by,

$$
\mathrm{F}(\mathrm{q})=\exp \{-\operatorname{xp}(-[\mathrm{q}-\mu] / \sigma)\}
$$

was fitted to the TSRs of $\mathrm{Q}_{m}$. The parameters $\mu$ and $\sigma$ are respectively the location and scale parameters. $q$ is the discharge magnitude. $\mu+0.557 \sigma$, $\mu$ and $1.283 \sigma$ are respectively the mean, mode and standard deviation of the distribution.

The results of Gumbel analysis of a typical experiment of test procedure 3 is summarized in Table 2. From these results, it is clear that mean and mode of the distribution are less than $30 \mathrm{pC}$ which is about one-fifth of that observed after the start of burst discharges. Also the scatter in the magnitudes of $q$ were substantially higher as brought out by the column for standard deviation in Table 2. Similar results were obtained for other test procedures.

Post-burst PD data was observed to follow a twoparameter Weibull distribution given by,

$$
\mathrm{F}(\mathrm{q})=1-\exp ((-\mathrm{q}-\gamma) / \alpha)^{\beta}
$$

where $\alpha$ is the scale parameter which represents the 63.2 percentile of $\mathrm{q}$. $\beta$ is a shape parameter. $\beta<1$ implies that insulation is improving; $\beta>1$ implies that insulation is ageing and higher the value of $\beta$, lesser is the life of the insulation.

Except for $\Phi_{7}$ and $\Phi_{8}$, it is a clear three-mode Weibull fit for all other phasies and that of full cycle. However for the purpose of analysis only the last mode have been considered for analysis as their values of $\alpha$ and $\beta$ are much higher than their corresponding values of the previous modes. In case of $\Phi_{7}$ and $\Phi_{8}$ a single mode Weibull fits the PD data.

The $\alpha$ and $\beta$ ranges of cycle TSRs of test procedures 1 to 3 are as follows:

\begin{tabular}{lrr} 
& $\alpha$-range & \multicolumn{2}{c}{$\beta$-range } \\
Proc. \#1 & 80 to $95 \mathrm{pC}$ & 4 to 8 \\
Proc. \#2 & 85 to $100 \mathrm{pC}$ & 4 to 9 \\
Proc. \#3 & 110 to $120 \mathrm{pC}$ & 12 to 20
\end{tabular}

It is to be noted that while $\alpha$ and $\beta$ of $\Phi_{14}$ and/or $\Phi_{13}$ closely match with the above values, those that of $\Phi_{7}$ and $\Phi_{8}$ were only a third or half that of their corresponding cycle values.

TSRs of $\alpha, \beta, \mathrm{N}_{t}$ and $\mathrm{Q}_{t}$ were obtained as before and Spearman's rank correlattion test statistic [3] was used to study the degree of correlation between Weibull and other PD related quantities of each phase and that of full cycle.

The correlation results of aging experiments of test procedure 2 and 3 are given in Tables 3 and 4. Correlation results of experiments of test procedure 1 were similar to that of test procedure 2 [4]. The significance level $(\sigma)$ of the results presented in the table is less than 0.2 , except for $\Phi_{14}$ where $\sigma \leq 0.1$. Blanks $(-)$ in the table means that there is no correlation at $\sigma \leq 0.2$.

Correlation results $\alpha_{p h}$ to $\alpha_{c y}, \beta_{p h}$ to $\beta_{c y}$ compare the aging process, as monitored by Weibull parameters $\alpha$ and $\beta$, in individual phases (ph) with the corresponding process of the full cycle (cy). As the aging progresses, depending upon its intensity the values of $\mathrm{N}_{t}$ and $\mathrm{Q}_{t}$ also vary accordingly. The trend of these variations in individual phases is compared with the trend of the full cycle using phase to cycle correlations of $N_{t}$ and $Q_{t}\left(N_{p h}\right.$ to $N_{c y}, Q_{p h}$ to $\mathrm{Q}_{c y}$ ). In a similar manner, other correlation parameters like $\alpha_{p h}$ to $\mathrm{N}_{p h}, \alpha_{p h}$ to $\mathrm{Q}_{p h}, \beta_{p h}$ to $\mathrm{N}_{p h}$ and $\beta_{p h}$ to $Q_{p h}$ relate the phase values of Weibull's $\alpha$ and $\beta$ with their corresponding values of $N_{t}$ and $Q_{t}$.

A strong positive correlation is seen between all correlation parameters in $\Phi_{14}$ and $\Phi_{13}$. However the 
observation regarding $\Phi_{13}$ is true only for test procedure 3 . Correlation between phase to cycle quantities of Weibull's $\alpha$ and $\beta$ shows that for all procedures, $\Phi_{14}$ records the highest positive value of correlation coefficient $(\rho)$ and this is followed by either $\Phi_{16}$ for procedure $1 \& 2$ or $\Phi_{13}$ for procedure 3 . It can also be seen that no other phase records a positive value of $\rho$ comparable to the above mentioned phases.

A strong positive correlation observed in these phases only implies that the aging mechanism of this phase closely matches with that of the corresponding full cycle process. This is further augmented by the earlier observation made in the preceding section that $\alpha$ and $\beta$ of $\Phi_{14}$ and/or $\Phi_{13}$ closely matches that of $\Phi_{c y}$.

In general a good positive correlation is seen for all phases between $\mathrm{N}_{p h}$ to $\mathrm{N}_{c y}, \mathrm{Q}_{p h}$ to $\mathrm{Q}_{c y}$ and $\mathrm{N}_{p h}$ to $Q_{p h}$ this may be explained by the tendency of phase values of $N_{t}$ and $Q_{t}$ to increase with aging time.

\section{Breakdown Mechanism and Model}

A visual examination of failed sample reveal the presence of marks of discharges tracing a circular ring like path around the contact point of the sample surface with the electrodes. The breakdown occurred on the circumference of this path. As pointed out by Takuma [5] the field enhancements in these wedge shaped zones can be as high as 300 percent for $\epsilon_{\text {solid }} / \epsilon_{\text {liquid }}$ ratio of 2 . It is this field that contribute to the generation of a streamer which plays a important role in causing breakdown. If the streamers reaching the sample surface is assumed as a conductor and space charge generation ignored then it can be regarded as a metal pin in contact with the sample surface. It was pointed out by Mizuno [6] that internal field distribution in the sample rather than at the streamer tip plays a role in breakdown. If the electric field beneath the streamer tip attenuates rapidly into the sample thickness then streamer does not penetrate deep into the sample and hence surface degradation instead of breakdown only takes place. On the other hand if the field in the sample beneath the streamer tip is sufficiently high to support the development into it, then breakdown occurrs.

\section{Conclusions}

Irrespective of test procedure, stress level or its duration, a common $\mathrm{PD}$ signature was observed for this insulation configuration. Two phases $\Phi_{13}$ and $\Phi_{14}$ near the vicinity of the negative voltage top was found to contribute significantly to the full cycle aging process. The aging mechanism of these phases were also found to correlate well with that of their corresponding cycle process.

\section{References}

1. Raja, K. Narayanachar, M.N. and Nema, R.S "Phase Resolved Partial Discharge Measuring System for Insulation Studies", Journal of the Instrumentation Society of India, Vol.25, No. 3\&4, 1995.

2. Hahn, G.J. and Shapiro, S.S. Statistical Models in Engineering, John Wiley \& Sons, New York, 1967.

3. Conover, W.J. Practical Non-parametric Statistics, John Wiley \& Sons, New York, 1980.

4. Raja, K. Narayanachar, M.N. and Nema, R.S. "A Study of Phase Angle Distribution of Partial Discharges in Oil-Pressboard Insulation Systems", 1996 IEEE International Symposium on Electrical Insulation, Paper No. 2A-14.

5. Takuma, T and Kawamoto, T. "Field Intensification Near Various Points of Contact with a Zero Contact Angle Between a Solid Dielectric and an Electrode", IEEE Trans. on PAS. Vol. PAS-163, No. 9, Sept. 1984, pp. 2186-2494.

6. Mizuno, Y. Tsuge, T. Shimizu, N. and Horii, K. "A Study of Electric Breakdown of Polymer Film by Partial Discharge in Liquid Dielectrics", Electrical Engineering In Japan, Vol. 104, No. 2, 1984, pp. 19-25.

Table 1: Phase Percentages of Full Cycle $N_{t}$

\begin{tabular}{|c|c|c|c|c|c|c|c|c|c|c|c|c|c|c|c|c|}
\hline Proc \# & $\Phi_{1}$ & $\Phi_{2}$ & $\Phi_{3}$ & $\Phi_{4}$ & $\Phi_{5}$ & $\Phi_{6}$ & $\Phi_{7}$ & $\Phi_{8}$ & $\Phi_{9}$ & $\Phi_{10}$ & $\Phi_{11}$ & $\Phi_{12}$ & $\Phi_{13}$ & $\Phi_{14}$ & $\Phi_{15}$ & $\Phi_{16}$ \\
\hline \hline 1 & 2.99 & 3.43 & 3.36 & 3.55 & 2.05 & 2.24 & 0.66 & 1.01 & 2.64 & 3.68 & 4.66 & 5.09 & 5.11 & 38.06 & 3.39 & 17.99 \\
2 & 2.67 & 2.38 & 2.24 & 3.52 & 1.61 & 1.59 & 0.40 & 0.90 & 2.36 & 3.66 & 3.73 & 4.00 & 5.28 & 38.71 & 5.32 & 21.65 \\
3 & 3.47 & 3.82 & 4.70 & 4.53 & 2.32 & 2.58 & 0.39 & 0.92 & 4.54 & 5.17 & 6.55 & 7.42 & 12.33 & 32.20 & 2.39 & 6.66 \\
\hline \hline
\end{tabular}


Table 2: Results of Gumbel Analysis of a Typical Experiment of Test Procedure 3

\begin{tabular}{|c|c|c|c|c|c|c|c|c|c|c|c|c|c|c|c|c|c|}
\hline $\begin{array}{l}\text { Gumbel \& } \\
\text { Derived } \\
\text { Parameters }\end{array}$ & $\Phi_{1}$ & $\Phi_{2}$ & $\Phi_{3}$ & $\Phi_{4}$ & $\Phi_{5}$ & $\Phi_{6}$ & $\Phi_{7}$ & $\Phi_{8}$ & $\Phi_{9}$ & $\Phi_{10}$ & $\Phi_{11}$ & $\Phi_{12}$ & $\Phi_{13}$ & $\boldsymbol{\Phi}_{144}$ & $\Phi_{15}$ & $\Phi_{16}$ & $\Phi_{c y}$ \\
\hline$\overline{\text { Scale }}$ & 11.3 & 11.2 & 10.1 & 6.1 & 9.6 & 3.1 & 7.1 & $\overline{5.8}$ & $\mid 10.7$ & 11.2 & 7.6 & 7.2 & 8.3 & 7.3 & 4.9 & 2.6 & 11.2 \\
\hline Location & 24.9 & 24.4 & 235 & 23.3 & 21.4 & 15.7 & 12.8 & 13.8 & 24.9 & 22.7 & 23.5 & 25.0 & 26.3 & 21.0 & 17.1 & 18.0 & 33.2 \\
\hline Mean & 31.5 & 30.9 & 29.4 & 26.9 & 26.9 & 17.5 & 16.9 & 17.2 & 31.2 & 29.2 & 27.8 & 29.1 & 31.1 & 25.2 & 19.9 & 19.5 & 32.7 \\
\hline Mode & 25.0 & 24.4 & 23.5 & 23.4 & 21.4 & 15.7 & 12.8 & 13.8 & 24.8 & 22.7 & 23.5 & 25.0 & 26.3 & 21.0 & 17.1 & 18.0 & 33.2 \\
\hline Standard & & & & & & & & & & & & & & & & & \\
\hline Deviation & 14.5 & 14.4 & 13.0 & 7.8 & 12.3 & 4.0 & 9.2 & 7.4 & 13.7 & 14.3 & 9.7 & 9.3 & 10.6 & 9.4 & 6.3 & 3.4 & 14. \\
\hline
\end{tabular}

Table 3: Correlation Results of a Typical Experiment of Test Procedure 2

\begin{tabular}{|l|c|c|c|c|c|c|c|c|c|c|c|c|c|c|c|c|}
\hline $\begin{array}{l}\text { Correlation } \\
\text { Parameter }\end{array}$ & $\Phi_{1}$ & $\boldsymbol{\Phi}_{2}$ & $\boldsymbol{\Phi}_{3}$ & $\boldsymbol{\Phi}_{4}$ & $\Phi_{5}$ & $\Phi_{6}$ & $\Phi_{7}$ & $\Phi_{8}$ & $\Phi_{9}$ & $\Phi_{10}$ & $\Phi_{11}$ & $\Phi_{12}$ & $\Phi_{13}$ & $\Phi_{14}$ & $\Phi_{15}$ & $\Phi_{16}$ \\
\hline \hline$\alpha_{p h}$ to $\alpha_{c y}$ & 0.57 & - & - & - & - & - & -0.52 & - & - & - & - & - & - & 0.98 & - & 0.73 \\
$\beta_{p h}$ to $\beta_{c y}$ & - & -0.60 & - & - & - & - & - & - & - & - & - & - & - & 0.73 & -0.47 & 0.65 \\
$N_{p h}$ to $N_{c y}$ & 0.83 & - & - & - & -0.57 & - & - & - & -0.68 & - & 0.85 & 0.78 & 0.78 & 1.00 & 0.87 & 0.98 \\
$Q_{p h}$ to $Q_{c y}$ & 0.68 & - & - & 0.63 & - & -0.47 & - & - & - & - & 0.72 & 0.47 & 0.62 & 0.82 & 0.78 & 0.83 \\
$\alpha_{p h}$ to $N_{p h}$ & 0.57 & - & -0.52 & - & - & - & - & 0.57 & 0.67 & - & - & 0.84 & - & 0.90 & 0.50 & 0.65 \\
$\alpha_{p h}$ to $Q_{p h}$ & - & - & -0.83 & - & - & -0.65 & - & 0.83 & - & - & - & 0.90 & - & 0.87 & 0.52 & 0.70 \\
$\beta_{p h}$ to $N_{p h}$ & 0.50 & - & - & - & - & - & - & - & 0.63 & - & - & - & - & 0.92 & - & 0.53 \\
$\beta_{p h}$ to $Q_{p h}$ & - & - & - & - & - & 0.67 & - & - & - & 0.45 & - & 0.45 & - & 0.95 & - & 0.55 \\
$\alpha_{p h}$ to $\beta_{p h}$ & - & - & - & 0.85 & 0.90 & - & - & - & - & 0.60 & -0.62 & 0.53 & - & 0.82 & - & - \\
$N_{p h}$ to $Q_{p h}$ & 0.72 & 0.83 & 0.83 & - & - & - & 0.88 & 0.80 & 0.87 & 0.65 & 0.87 & 0.90 & 0.93 & 0.98 & 0.95 & 0.98 \\
\hline
\end{tabular}

Table 4: Correlation Results of a Typical Experiment of Test Procedure 3

\begin{tabular}{|l|c|c|c|c|c|c|c|c|c|c|c|c|c|c|c|c|}
\hline $\begin{array}{l}\text { Correlation } \\
\text { Parameter }\end{array}$ & $\Phi_{1}$ & $\Phi_{2}$ & $\Phi_{3}$ & $\Phi_{4}$ & $\Phi_{5}$ & $\Phi_{6}$ & $\Phi_{7}$ & $\Phi_{8}$ & $\Phi_{9}$ & $\Phi_{10}$ & $\Phi_{11}$ & $\Phi_{12}$ & $\Phi_{13}$ & $\Phi_{14}$ & $\Phi_{15}$ & $\Phi_{16}$ \\
\hline \hline$\alpha_{p h}$ to $\alpha_{c y}$ & - & - & - & - & - & -0.42 & - & - & - & - & - & -0.51 & 0.66 & 0.82 & - & - \\
$\beta_{p h}$ to $\beta_{c y}$ & - & - & - & - & -0.49 & -0.45 & - & - & - & - & 0.53 & - & 0.86 & 0.86 & - & - \\
$N_{p h}$ to $N_{c y}$ & - & - & - & - & - & - & - & - & 0.39 & - & - & 0.53 & 0.80 & 0.85 & - & 0.63 \\
$Q_{p h}$ to $Q_{c y}$ & 0.39 & 0.66 & 0.59 & 0.83 & 0.57 & 0.76 & 0.46 & - & - & 0.64 & - & 0.54 & 0.76 & 0.78 & 0.39 & - \\
$\alpha_{p h}$ to $N_{p h}$ & - & - & 0.42 & 0.50 & 0.76 & 0.49 & 0.57 & - & -0.41 & - & - & -0.43 & 0.84 & 0.79 & - & - \\
$\alpha_{p h}$ to $Q_{p h}$ & 0.63 & - & 0.46 & 0.71 & - & 0.76 & 0.61 & - & - & - & - & - & 0.81 & 0.78 & - & - \\
$\beta_{p h}$ to $N_{p h}$ & - & - & - & - & 0.46 & - & - & 0.45 & - & - & - & - & 0.73 & 0.70 & - & - \\
$\beta_{p h}$ to $Q_{p h}$ & - & - & - & - & 0.57 & 0.74 & - & 0.57 & - & - & - & - & 0.74 & 0.73 & - & - \\
$\alpha_{p h}$ to $\beta_{p h}$ & 0.49 & - & - & - & 0.80 & 0.89 & 0.43 & - & 0.48 & - & 0.41 & - & 0.61 & 0.48 & 0.75 & 0.80 \\
$N_{p h}$ to $Q_{p h}$ & 0.56 & 0.97 & 0.97 & 0.87 & 0.87 & 0.83 & 0.97 & 0.95 & 0.97 & 0.56 & 0.98 & 0.64 & 0.94 & 0.98 & 0.87 & 0.90 \\
\hline \hline
\end{tabular}

